\title{
Measuring senescence rates of patients with end-stage renal disease while accounting for population heterogeneity : an analysis of data from the ERA-EDTA Registry
}

Koopman, Jacob J. E.

2016-11

Koopman , J J E , Kramer , A , van Heemst , D , Asberg , A , Beuscart , J-B , Buturovic-Ponikvar , J , Collart , F , Couchoud , C G , Finne , P , Heaf , J G, Massy , Z A , De Meester, J M J , Palsson, R, Steenkamp, R, Traynor , J P , Jager , K J \& Putter , H 2016 , ' Measuring senescence rates of patients with end-stage renal disease while accounting for population heterogeneity : an analysis of data from the ERA-EDTA Registry ' , Annals of Epidemiology, vol. 26 , no. 11 , pp. 773-779 . https://doi.org/10.1016/j.annepidem.2016.08.010

http://hdl.handle.net/10138/229658

https://doi.org/10.1016/j.annepidem.2016.08.010

publishedVersion

Downloaded from Helda, University of Helsinki institutional repository.

This is an electronic reprint of the original article.

This reprint may differ from the original in pagination and typographic detail.

Please cite the original version. 
Original article

\section{Measuring senescence rates of patients with end-stage renal disease while accounting for population heterogeneity: an analysis of data from the ERA-EDTA Registry}

Jacob J.E. Koopman MD, PhD ${ }^{\mathrm{a}, *}$, Anneke Kramer PhD ${ }^{\mathrm{b}}$, Diana van Heemst PhD ${ }^{\mathrm{a}}$, Anders Åsberg PhD ${ }^{\mathrm{c}}$, Jean-Baptiste Beuscart MD, PhD ${ }^{\mathrm{d}, \mathrm{e}}$, Jadranka Buturović-Ponikvar MD, PhD ${ }^{\mathrm{f}, \mathrm{g}}$, Frederic Collart $\mathrm{MD}, \mathrm{PhD}^{\mathrm{h}}$, Cécile G. Couchoud MD, PhD ${ }^{\mathrm{i}}$, Patrik Finne MD, PhD ${ }^{\mathrm{j}, \mathrm{k}}$, James G. Heaf MD, DMSc ${ }^{1}$, Ziad A. Massy MD ${ }^{\mathrm{m}, \mathrm{n}}$, Johan M.J. De Meester MD, PhD ${ }^{\circ}$, Runolfur Palsson MD ${ }^{\mathrm{p}, \mathrm{q}}$, Retha Steenkamp PhD ${ }^{\mathrm{r}}$, Jamie P. Traynor MD ${ }^{\mathrm{s}}$, Kitty J. Jager MD, $\mathrm{PhD}^{\mathrm{b}}$, Hein Putter $\mathrm{PhD}^{\mathrm{t}}$

${ }^{a}$ Section of Gerontology and Geriatrics, Department of Internal Medicine, Leiden University Medical Center, Leiden, The Netherlands

${ }^{\mathrm{b}}$ ERA-EDTA Registry, Department of Medical Informatics, Academic Medical Center, University of Amsterdam, Amsterdam, The Netherlands

${ }^{\mathrm{c}}$ Norwegian Renal Registry, Department of Transplant Medicine, Oslo University Hospital-Rikshospitalet, Oslo, Norway

${ }^{\mathrm{d}}$ University of Lille, EA2694, Santé publique: épidémiologie et qualité des soins, Lille, France

${ }^{\text {e }} \mathrm{CHU}$ Lille, Geriatric Department, Lille, France

${ }_{\mathrm{f}}^{\mathrm{f}}$ Department of Nephrology, Ljubljana University Medical Center, Ljubljana, Slovenia

${ }^{\mathrm{g}}$ Medical Faculty, University of Ljubljana, Ljubljana, Slovenia

${ }^{\mathrm{h}}$ Department of Nephrology and Dialysis, Brugmann University Hospital, Brussels, Belgium

${ }^{\mathrm{i}}$ Renal Epidemiology and Information Network (REIN) Registry, French Biomedical Agency, Saint-Denis-la-Plaine, France

${ }^{\mathrm{j}}$ Finnish Registry for Kidney Diseases, Helsinki, Finland

${ }^{\mathrm{k}}$ Department of Nephrology, Helsinki University Central Hospital, Helsinki, Finland

${ }^{1}$ Department of Medicine, Zealand University Hospital, Roskilde, Denmark

${ }^{\mathrm{m}}$ Division of Nephrology, Ambroise Paré University Hospital, University of Paris Ouest-Versailles-St-Quentin-en-Yvelines, Paris, France

${ }^{\mathrm{n}}$ Institut National de la Santé et de la Recherche Médicale (INSERM) U1018, Research Centre in Epidemiology and Population Health (CESP), Villejuif, France

${ }^{\circ}$ Department of Nephrology, Dialysis, and Hypertension, AZ Nikolaas, Sint-Niklaas, Belgium

${ }^{\mathrm{p}}$ Division of Nephrology, Landspitali-The National University Hospital of Iceland, Reykjavik, Iceland

${ }^{\mathrm{q}}$ Faculty of Medicine, School of Health Sciences, University of Iceland, Reykjavik, Iceland

${ }^{\mathrm{r}}$ UK Renal Registry, Southmead Hospital, Bristol, UK

${ }^{\mathrm{s}}$ The Scottish Renal Registry, Information Services Division Scotland, Glasgow, UK

${ }^{\mathrm{t}}$ Department of Medical Statistics and Bioinformatics, Leiden University Medical Center, Leiden, The Netherlands

\section{A R T I C L E I N F O}

Article history:

Received 7 April 2016

Accepted 23 August 2016

Available online 31 August 2016

\section{Keywords:}

Aging

Dialysis

End-stage renal disease

Frailty

Gompertz model

Kidney transplantation

Mortality rate

Population heterogeneity

Senescence

Senescence rate

\begin{abstract}
A B S T R A C T
Purpose: Although a population's senescence rate is classically measured as the increase in mortality rate with age on a logarithmic scale, it may be more accurately measured as the increase on a linear scale. Patients on dialysis, who suffer from accelerated senescence, exhibit a smaller increase in their mortality rate on a logarithmic scale, but a larger increase on a linear scale than patients with a functioning kidney transplant. However, this comparison may be biased by population heterogeneity.

Methods: Follow-up data on 323,308 patients on dialysis and 91,679 patients with a functioning kidney transplant were derived from the ERA-EDTA Registry. We measured the increases in their mortality rates using Gompertz frailty models that allow individual variation in this increase.

Results: According to these models, the senescence rate measured as the increase in mortality rate on a logarithmic scale was smaller in patients on dialysis, while the senescence rate measured as the increase on a linear scale was larger in patients on dialysis than patients with a functioning kidney transplant. Conclusions: Also when accounting for population heterogeneity, a population's senescence rate is more accurately measured as the increase in mortality rate on a linear scale than a logarithmic scale.
\end{abstract}

(c) 2016 Elsevier Inc. All rights reserved.

The authors declare no conflicts of interest.

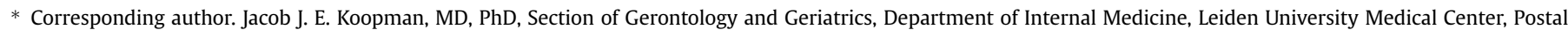
Zone C7-Q, PO Box 9600, 2300 RC Leiden, The Netherlands. Tel.: +31-71-5266640; fax: +31-71-5266912.

E-mail address: j.j.e.koopman@lumc.nl (J.J.E. Koopman). 


\section{Introduction}

Senescence is constituted by a complex of biological mechanisms that lead to an increase in vulnerability to death from adolescence onward. A population's senescence rate can consequently be measured as an increase in mortality rate with age [1-3]. Classically, increases in mortality rates are measured on a logarithmic scale, which can be done using the Gompertz model [1-3]. However, it has been argued that the increase in mortality rate on a logarithmic scale is an inaccurate measure of a population's senescence rate [4]. Instead, it has been proposed that the senescence rate should be measured as the increase in mortality rate on a linear scale, which can also be determined using the Gompertz model [5]. This proposition has been empirically tested in patients with end-stage renal disease on dialysis and with a functioning kidney transplant [6]. Patients on dialysis are generally acknowledged to suffer both clinically and biologically from accelerated senescence. They exhibit higher prevalences and more rapid progression of various age-related disorders as compared with the general population. After kidney transplantation, these phenomena of accelerated senescence improve [6-9]. When measured as the increases in their mortality rates on a logarithmic scale, patients on dialysis have a lower senescence rate than patients with a functioning transplant. By contrast, when measured as the increases in their mortality rates on a linear scale, patients on dialysis have a higher senescence rate than patients with a functioning transplant [6]. These findings support the proposition that a senescence rate measured as the increase in mortality rate on a linear scale corresponds more accurately with clinical and biological knowledge than a senescence rate measured as the increase in mortality rate on a logarithmic scale.

Previous comparisons of both methods may have been biased because they have not accounted for population heterogeneity. Most populations are heterogeneous as they consist of individuals with different levels of frailty [10-12]. Distinctive from the clinical diagnosis of frailty, all individuals can be attributed a level of frailty in this statistical-epidemiological sense. At any age, individuals with higher levels of frailty suffer from an elevated mortality rate as compared with less frail individuals. In patients with end-stage renal disease, differences in frailty may result from varieties in primary renal disease, treatment modality, quality of care, ethnicity, and country [13-17]. Heterogeneity among patients on dialysis is likely greater than among patients with a functioning transplant because the more healthy patients are considered eligible for transplantation [13-15]. Subpopulations with different levels of frailty may display age-dependent increases in mortality rates that are different as well as different from the increase observed in the population as a whole [10-12]. Consequently, a senescence rate measured as the increase in mortality rate with age in a population as a whole may not correctly represent the senescence rates of the different subpopulations.

Extensions of models like the Gompertz model have been developed to account for population heterogeneity [10-12], but these models have not yet been used for comparing both methods to measure senescence rates. These models contain a frailty parameter that allows individual variation in the age-dependent increase in mortality rate. Here, we use such Gompertz frailty models to measure the senescence rates of patients with end-stage renal disease on dialysis and with a functioning kidney transplant while accounting for population heterogeneity. Now uncovering the possible bias due to population heterogeneity, we aim to compare the senescence rates measured as the increases in their mortality rates on a logarithmic scale, according to the classical method, and measured as the increases in their mortality rates on a linear scale, according to the alternative method.

\section{Methods}

\section{Study population}

This study included data from 27 national and regional registries (see Appendix A) participating in the Registry of the European Renal Association-European Dialysis and Transplant Association. These registries record the treatment and survival history of European patients receiving renal replacement therapy, either dialysis or kidney transplantation [15]. Patients were included if renal replacement therapy was started in or after 1985; follow-up ended on January 1, 2013. At baseline, the country or region of origin, birth date, sex, and primary renal disease were registered for each patient. Countries or regions of origin were grouped into four categories: Western Europe, Northern Europe, Southeastern Europe, and Southwestern Europe (see Appendix A). Primary renal diseases were also grouped into four categories: glomerular diseases including glomerulonephritis and glomerulosclerosis, pyelonephritis, vascular diseases including renal diseases due to diabetes mellitus, hypertension, and vascular disease and other or unknown diseases.

\section{Follow-up and crude mortality rates}

During follow-up, the dates and modalities of renal replacement therapy and the date of death were collected for each patient from the age of 20 years onward. For patients on dialysis, follow-up started 6 months after initiation of dialysis, excluding early mortality related to dialysis treatment or preceding transplantation, and lasted until death, transplantation, recovery of renal function, loss to follow-up, or January 1, 2013. The type of dialysis was categorized as hemodialysis or peritoneal dialysis. For patients with a functioning transplant, follow-up started 6 months after transplantation, excluding early mortality related to surgery or preceding dialysis treatment, and lasted until death, transfer to dialysis due to transplant failure, loss to follow-up, or January 1, 2013. Each transplantation was categorized according to the type of donor, being a deceased donor, living donor, or unknown donor.

Crude age-specific mortality rates were calculated from the follow-up data of the individual patients without using the Gompertz model by dividing the number of deaths by the number of personyears of follow-up per 5-year age group. Confidence intervals of the mortality rates were calculated using Byar's formula [18].

\section{Mortality rates determined by the Gompertz models}

The Gompertz model is described by $m(t)=\alpha \mathrm{e}^{\gamma t}$, where $m(t)$ is the mortality rate at age $t$ and $\alpha$ and $\gamma$ the model's parameters. We used two Gompertz models that accounted for population heterogeneity. Both models were adjusted for covariates that affect the mortality rates, including the patient's sex, country of origin, primary renal disease, type of dialysis, and type of kidney donor. In addition, each model contained a random frailty parameter $z$ that allowed further individual variation in the mortality rates through variation in either of the Gompertz model's parameters $\alpha$ and $\gamma$. Variation in $\alpha$ multiplies mortality rates at all ages by an ageindependent factor; variation in $\gamma$ multiplies mortality rates by a factor that increases with age. The Gompertz frailty models were fitted to the follow-up data by numerically integrating the frailty parameter $z$ and numerically maximizing the log-likelihood with respect to $\alpha, \gamma$, and $z$, as explained in more detail in Appendix A.

\section{Senescence rates determined by the Gompertz models}

We determined the senescence rates of the patients using the two Gompertz frailty models. The senescence rates measured as the 
increases in their mortality rates on a logarithmic scale, according to the classical method, are determined by the derivative functions of the logarithmically transformed models. The senescence rates measured as the increases in their mortality rates on a linear scale, according to the alternative method, are determined by the derivative function of the model without logarithmic transformation. The derivative functions of the Gompertz frailty models are given in Appendix A.

The Gompertz frailty models were fitted, and mortality rates and senescence rates were measured by these models using $R$, version 3.0 (R Foundation for Statistical Computing, Vienna, Austria).

\section{Results}

Table 1 describes the characteristics of the study population. Because patients could undergo both dialysis treatment and kidney transplantation, 60,817 patients (17.2\%) contributed 575,858 person-years of follow-up (34.6\%) to both treatment groups. The maximum number of a patient's treatments of dialysis and transplantation was 14 years.

Figure 1 shows the age-specific mortality rates of patients on dialysis and patients with a functioning transplant. The crude mortality rates calculated without using the Gompertz model are indicated in gray. At all ages, mortality rates were higher in patients on dialysis than patients with a functioning transplant. On a linear scale (Fig. 1A and 1B), the mortality rates increased exponentially but leveled in patients on dialysis and declined in patients with a functioning transplant after the age of 85-90 years, whereas the confidence intervals widened after these ages due to decreasing numbers of patients. On a logarithmic scale (Fig. 1C and 1D), the exponential increases in the mortality rates conformed to a linear increase and their leveling and decline were discernible as a deviation from the linear increase.

We fitted two Gompertz frailty models to the follow-up data of patients on dialysis and patients with a functioning transplant: one with a frailty parameter $z$ allowing individual variation in the model's parameter $\alpha$ and one with a frailty parameter $z$ allowing individual variation in the model's parameter $\gamma$. The obtained values for the models' parameters are given in Table 2 .

In Figure 1, the mortality rates of patients on dialysis and patients with a functioning transplant determined by the Gompertz frailty models are indicated in black. According to both models, the mortality rates were at all ages higher in patients on dialysis than patients with a functioning transplant. The mortality rates deviated from an exponential increase at the highest ages in both patient groups, deviating more according to the Gompertz frailty model with variation in $\gamma$ than the Gompertz frailty model with variation in $\alpha$. The mortality rates determined by the Gompertz frailty model with variation in $\alpha$ corresponded better with the crude mortality rates than those determined by the Gompertz frailty model with variation in $\gamma$ in patients on dialysis. At the highest ages, the mortality rates as determined by the Gompertz frailty model with variation in $\gamma$ corresponded better, but not well, with the leveling or declining crude mortality rates in both patients groups.

Figure 2 shows the age-specific senescence rates of patients on dialysis and patients with a functioning transplant. According to the classical method, the senescence rates were measured as the increases in their mortality rates on a logarithmic scale using the derivative functions of the logarithmically transformed Gompertz frailty models (Fig. 2A and 2B). These senescence rates were at all ages lower in patients on dialysis than patients with a functioning transplant according to both models. The difference in senescence rate between both patient groups was larger according to the Gompertz frailty model with variation in $\alpha$. The senescence rates were nearly constant according to the Gompertz frailty model with variation in $\alpha$ (Fig. 2A) but decreased in both patient groups according to the Gompertz frailty model with variation in $\gamma$ (Fig. 2B).

According to the alternative method, the senescence rates of patients on dialysis and patients with a functioning transplant were measured as the increases in their mortality rates on a linear scale using the derivative functions of the Gompertz frailty models without logarithmic transformation (Fig. 2C and 2D). According to both models, these senescence rates were at all ages higher in patients on dialysis than patients with a functioning transplant. The senescence rates increased nearly exponentially according to the

Table 1

Characteristics of the study population of patients with end-stage renal disease

\begin{tabular}{|c|c|c|c|}
\hline Characteristic & All patients & Patients on dialysis & Patients with a functioning kidney transplant \\
\hline \multicolumn{4}{|l|}{ At baseline } \\
\hline Patients, $n$ & 354,170 & 323,308 & 91,679 \\
\hline Males, $n(\%)$ & $217,864(61.5)$ & $198,611(61.4)$ & $57,433(62.6)$ \\
\hline Age at first treatment, median (IQR), y & $64.2(51.2-74.1)$ & $65.8(53.6-74.9)$ & $49.8(38.4-59.6)$ \\
\hline \multicolumn{4}{|l|}{ Region of origin, $n(\%)$} \\
\hline Western Europe & $136,873(38.6)$ & $125,873(38.9)$ & $37,515(40.9)$ \\
\hline Northern Europe & $45,307(12.8)$ & $38,910(12.0)$ & $15,999(17.5)$ \\
\hline Southeastern Europe & $42,426(12.0)$ & $41,373(12.8)$ & $4084(4.5)$ \\
\hline Southwestern Europe & $129,564(36.6)$ & $117,152(36.2)$ & $34,081(37.2)$ \\
\hline \multicolumn{4}{|l|}{ Primary renal disease, $n(\%)$} \\
\hline Glomerular diseases & $52,957(15.0)$ & $45,076(13.9)$ & $23,753(25.9)$ \\
\hline Pyelonephritis & $26,041(7.4)$ & $23,239(7.2)$ & $8251(9.0)$ \\
\hline Vascular diseases & $132,381(37.4)$ & $126,656(39.2)$ & $19,296(21.0)$ \\
\hline Other & $142,791(40.3)$ & $128,337(39.7)$ & $40,379(44.0)$ \\
\hline \multicolumn{4}{|l|}{ Follow-up } \\
\hline Total follow-up, person-years & $1,665,939$ & $1,049,987$ & 615,952 \\
\hline Follow-up per patient, median (IQR) person-years & $3.4(1.7-6.2)$ & $2.5(1.3-4.4)$ & $5.4(2.7-9.4)$ \\
\hline \multicolumn{4}{|l|}{ Dialysis modality, person-years (\%) } \\
\hline Hemodialysis & - & $901,710(85.9)$ & - \\
\hline Peritoneal dialysis & - & $148,278(14.1)$ & - \\
\hline \multicolumn{4}{|l|}{ Transplant source, person-years (\%) } \\
\hline Deceased donor & - & - & $471,799(76.6)$ \\
\hline Living donor & - & - & $115,512(18.8)$ \\
\hline Unknown & - & - & $28,641(4.6)$ \\
\hline Deaths, $n(\%)$ & $171,464(48.4)$ & $158,736(49.1)$ & $12,728(13.9)$ \\
\hline Age at death, median (IQR) years & $73.4(64.7-79.9)$ & $74.1(65.7-80.3)$ & $64.2(55.7-71.0)$ \\
\hline
\end{tabular}


Gompertz frailty model

with variation in $\alpha$

A

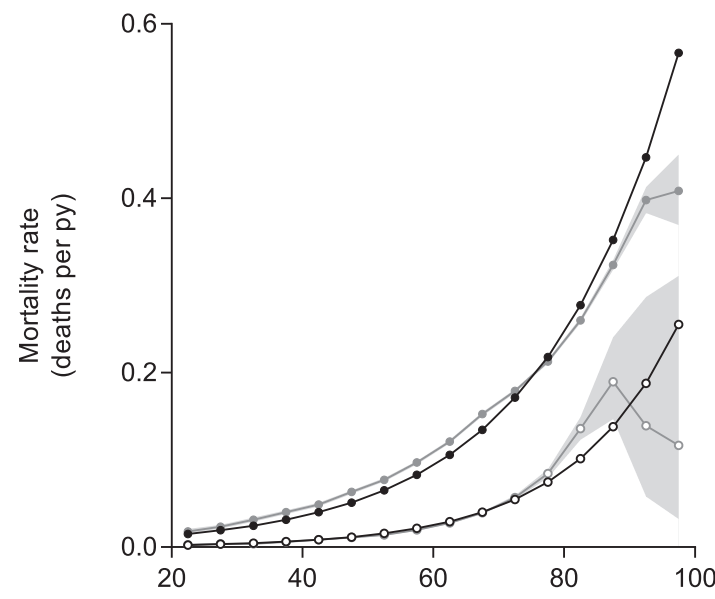

C

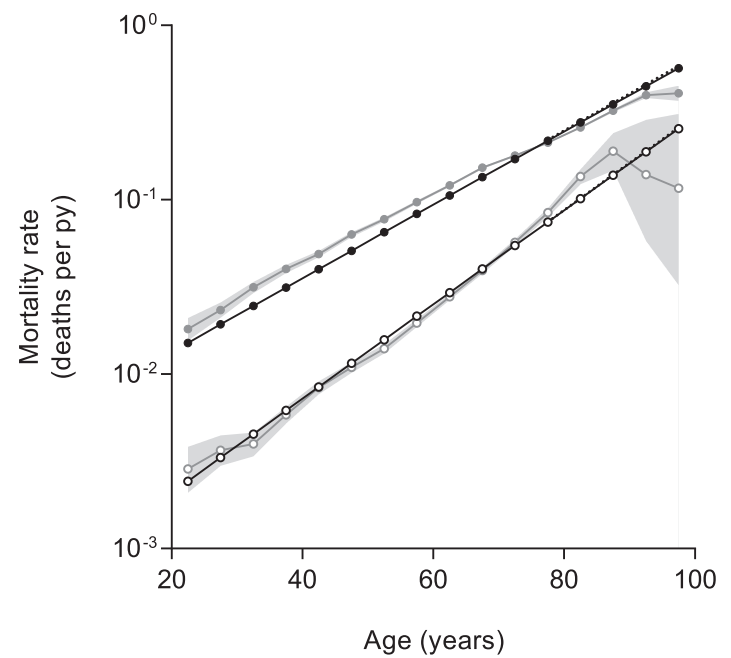

Gompertz frailty model

with variation in $\gamma$

B

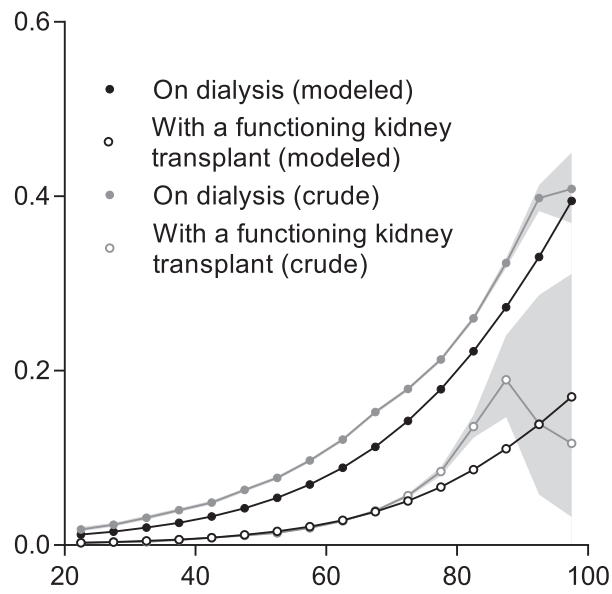

D

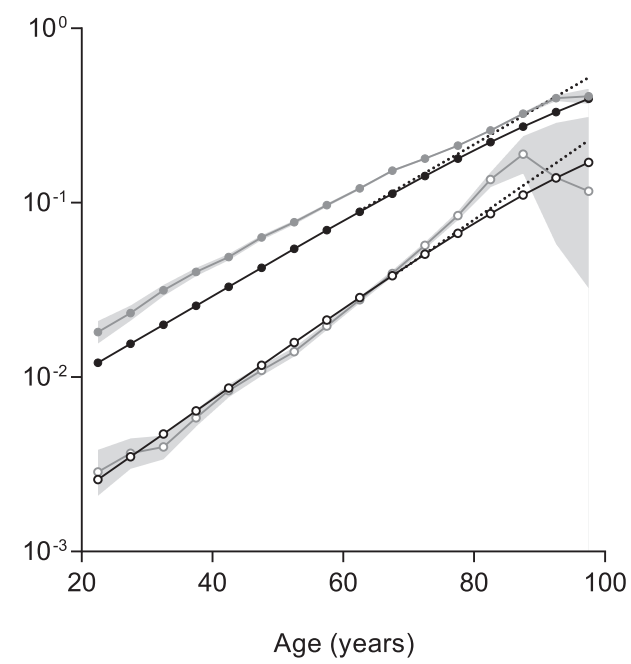

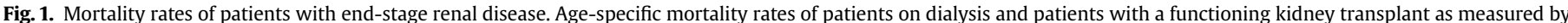

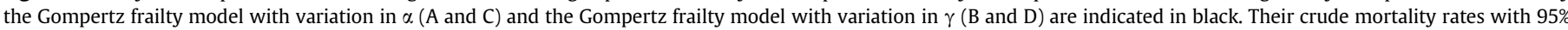

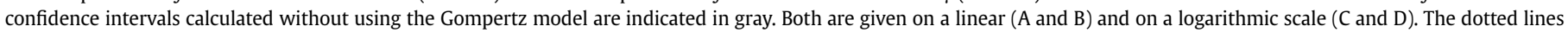
indicate linear increases on the logarithmic scale ( $C$ and $D)$. Py = person-years.

Gompertz frailty model with variation in $\alpha$ but deviated from an exponential increase and leveled at the highest ages in both patient groups according to the Gompertz frailty model with variation in $\gamma$. These senescence rates reached higher levels according to the Gompertz frailty model with variation in $\alpha$ than the Gompertz frailty model with variation in $\gamma$.

To visualize to what extent the increases in the mortality rates and senescence rates were allowed to vary by the Gompertz frailty models through variation in one of each model's parameters, the mortality rates and senescence rates for subpopulations with different levels of frailty are shown in Appendix B. The range covered by these subpopulations includes $95 \%$ of the patients. According to the Gompertz frailty model with variation in $\alpha, \alpha$ ranged from 0.45 to 0.56 in patients on dialysis and from 0.05 to 0.07 in patients with a functioning transplant. According to the Gompertz frailty model with variation in $\gamma, \gamma$ ranged from 4.59 to 5.49 in patients on dialysis and from 5.24 to 6.83 in patients with a functioning transplant. These parameters' values are given multiplied by 100 for readability. The variation in the age patterns was smaller according to the Gompertz frailty model with variation in $\alpha$ as compared with the Gompertz frailty model with variation in $\gamma$.

When repeating the analyses after excluding follow-up from the age of 85 years onward, similar results were obtained (data not shown).

\section{Discussion}

This study aims to compare the senescence rates of patients with end-stage renal disease on dialysis and with a functioning kidney transplant measured as the increases in their mortality rates on a logarithmic scale, according to the classical method, and measured as the increases in their mortality rates on a linear scale, according to an alternative method. Measured on a logarithmic scale, patients on dialysis had a lower senescence rate than patients with a functioning transplant. By contrast, measured on a linear scale, patients on dialysis had a higher senescence rate than patients with a functioning transplant. The alternative method, rather than the classical method, corresponds with the general knowledge that 
Table 2

Parameters of the Gompertz frailty models for patients with end-stage renal disease

\begin{tabular}{lll}
\hline Parameter & Patients on dialysis & $\begin{array}{l}\text { Patients with a functioning } \\
\text { kidney transplant }\end{array}$ \\
\hline $\begin{array}{l}\text { Gompertz frailty model } \\
\text { with variation in } \alpha\end{array}$ & \\
$\alpha \quad$ & $0.50(0.49-0.52)$ & $0.06(0.05-0.07)$ \\
$\gamma$ & $4.87(4.84-4.91)$ & $6.23(6.10-6.36)$ \\
$\sigma \quad$ & $5.27(4.51-6.15)$ & $6.94(3.95-12.20)$ \\
Gompertz frailty model & & \\
$\quad$ with variation in $\gamma$ & $0.39(0.38-0.40)$ & $0.07(0.06-0.07)$ \\
$\alpha \quad 5.02(5.01-5.04)$ & $5.98(5.91-6.05)$ \\
$\gamma \quad$ & $4.56(4.42-4.71)$ & $6.77(5.94-7.73)$ \\
$\sigma$ &
\end{tabular}

The values of the parameters of the Gompertz frailty models are given with $95 \%$ confidence intervals and multiplied by 100 for readability. The Gompertz model is described by parameter $\alpha$, which determines the minimal mortality rate at the youngest age, and by parameter $\gamma$, which determines the subsequent increase in mortality rate with age. The Gompertz frailty models have an additional frailty parameter $z$ that allows variation in either parameter $\alpha$ or $\gamma$ and has a normal distribution with a mean of 0 and a standard deviation of $\sigma$.

patients on dialysis suffer both clinically and biologically from accelerated senescence, whereas their senescence decelerates after transplantation [6-9].

Both methods to measure senescence rates have previously been compared in a smaller group of patients on dialysis and with a functioning kidney transplant, which is part of the population included in this study [6]. The results of this study are similar to the previous. As its novel contribution, this study extends the previous comparisons by using Gompertz frailty models that uncover possible bias due to population heterogeneity in two manners. Observed population heterogeneity was accounted for by adjusting the Gompertz model for known differences between subpopulations that affect the mortality rate, including the patient's sex, country of origin, primary renal disease, type of dialysis, and type of kidney donor. In addition, unobserved population heterogeneity was accounted for by adding a frailty parameter to the Gompertz model that allowed further individual variation in the increases in their mortality rates with age.

Moreover, we found that the senescence rates decreased with age according to the classical method, whereas they increased with age according to the alternative method. Increasing, rather than decreasing, senescence rates correspond with the general knowledge that older individuals exhibit higher prevalences and more rapid progression of various age-related disorders and a higher mortality rate as compared with younger individuals, also among patients with end-stage renal disease [1-3,5-9,13-16]. Together, these results reinforce the proposition that a population's senescence rate is more accurately measured as the increase in mortality rate on a linear scale than on a logarithmic scale, also when accounting for population heterogeneity.

We used one Gompertz frailty model with individual variation in the Gompertz model's parameter $\alpha$ and another one with individual variation in the Gompertz model's parameter $\gamma$. When $\alpha$ varies, mortality rates at all ages are multiplied by an ageindependent factor; the effect of variation in $\alpha$ is similar at all ages. When $\gamma$ varies, mortality rates are multiplied by a factor that increases with age; variation in $\gamma$ has a greater effect at higher ages. In line with this, we found that the increases in the mortality rates with age varied less according to the Gompertz frailty model with variation in $\alpha$ than the Gompertz frailty model with variation in $\gamma$ (Appendix B).

Mostly, the Gompertz frailty model with variation in $\alpha$ is used when the effects of population heterogeneity on the increases in mortality rates with age are studied [10-12]. This model explains leveling and declining mortality rates in several populations
[19-21]. Gompertz frailty models with variation in $\gamma$ have only recently been used and have been shown to explain leveling and declining mortality rates but have only rarely been applied to mortality data of human populations [22-25]. We found that the Gompertz frailty model with variation in $\gamma$ allowed the mortality rates to deviate more from an exponential increase and explained the leveling mortality rates better than the Gompertz frailty model with variation in $\alpha$.

A population's senescence rate can also be derived from the increase in mortality rate on a linear scale without using the Gompertz model [26]. Such a method enables the discovery of deviations from the exponential increases in mortality rate with age that are described by the Gompertz model. Leveling and declining mortality rates have been observed in populations at high ages [19,27], among which patients with end-stage renal disease [26]. It is presumed that such deviations may be effects of population heterogeneity, brought about by the joint study of subpopulations that have different levels of frailty [3,10-12]. When these subpopulations are studied separately, mortality rates increase exponentially up to the highest ages [28]. Consistent with this presumption, the Gompertz frailty models in this study that uncover population heterogeneity yielded leveling mortality rates. However, the Gompertz frailty models could not explain the observation of declining mortality rates. Other explanations than population heterogeneity can cause mortality rates to level and decline but are difficult to detect [22]. These other explanations may include the selection of relatively healthy patients for treatment at high age, the deceleration or cessation of senescence in individuals [22,27,29], and randomness or inaccuracy of the data [28].

More than estimating the senescence rates of patients on dialysis and patients with a functioning kidney transplant, of whom the former are generally known to age faster than the latter, this study reinforces the proposition that the alternative method to measure senescence rates is more accurate than the classical method. It shows that the conclusions based on both methods may even be opposite. It underscores, in general, the importance of verifying the validity of a method to measure the senescence rate of any population.

The classical method and the alternative method to measure senescence rates, which were compared in this study, represent two different definitions of the senescence rate. According to the classical method, the senescence rate is the relative increase in mortality rate with age, whereas according to the alternative method, it is the absolute increase in mortality rate. We have explained elsewhere how both definitions mathematically relate to each other and why they consequently may lead to opposite results [30].

It is essential for research on senescence to measure and compare senescence rates of populations. In humans, this is done to explore differences in senescence across time periods, environments, and ethnicities to reveal the influences on senescence of evolutionary, environmental, and genetic characteristics and to assess the effects on senescence of genetic and environmental risk factors and interventions [30]. The classical method is extensively used to measure senescence rates in research, but a reassessment using other methods, such as the alternative method studied here, may radically change previous conclusions. In the absence of agreement on the measurement of the senescence rate, one may resort to measuring an age-independent risk of mortality. However, a risk of mortality by itself does not reflect senescence; only the increase in this risk with age can be used to measure senescence [30]. One can choose to refrain from using models like the Gompertz model and to measure senescence rates nonparametrically as has been shown elsewhere [26]. Finally, one can measure a decline in health with age, which is a manifestation of senescence similar to the increase in vulnerability to death. Ideally, the narrow focus of 
Gompertz frailty model

with variation in $\alpha$

A
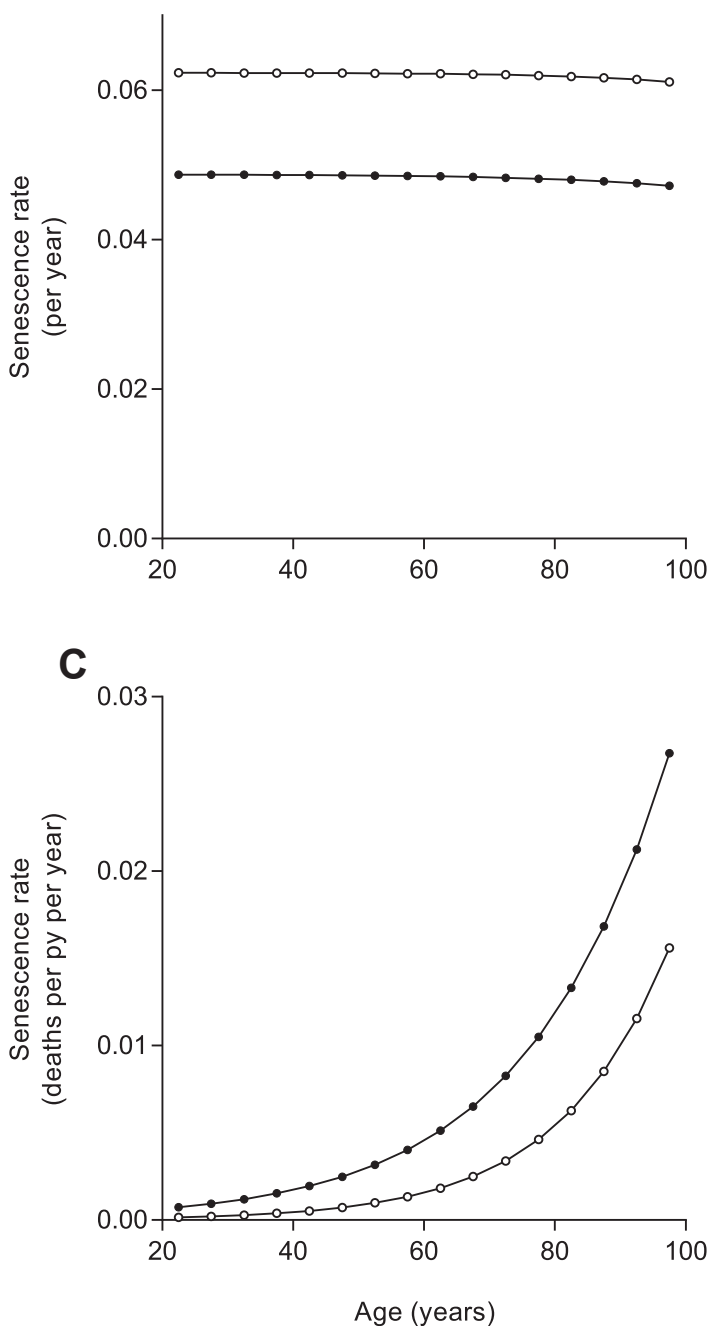

Gompertz frailty model

with variation in $\gamma$

B

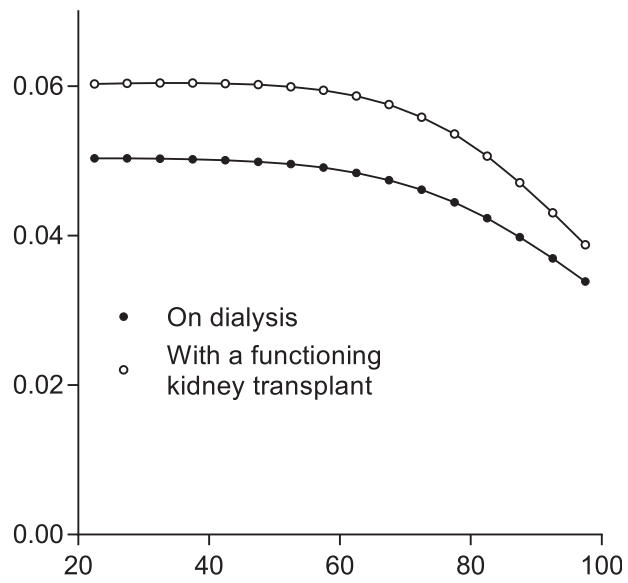

D

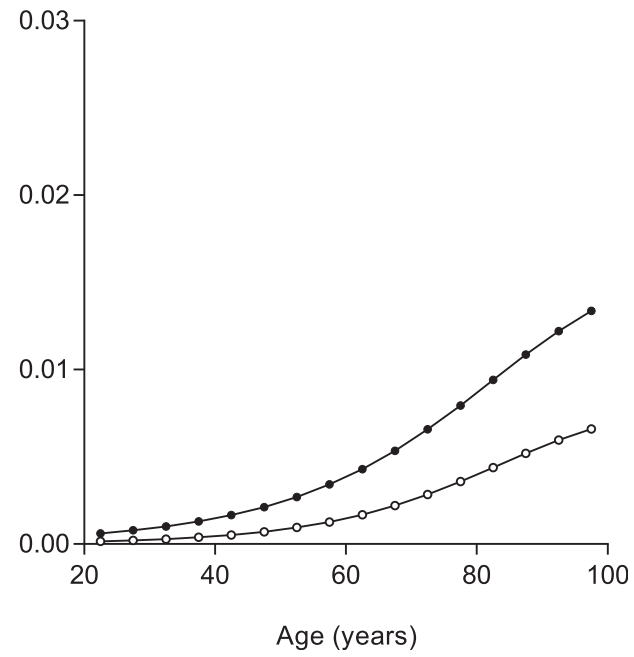

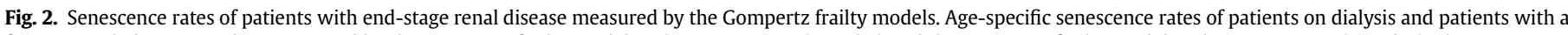

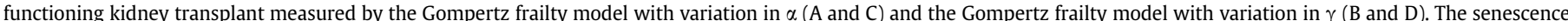

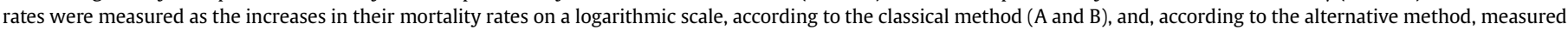
as the increases in their mortality rates on a linear scale (C and D). Py = person-years.

research on senescence using the classical method is complemented by a comparison with the use of these other methods.

In conclusion, a population's senescence rate is more accurately measured as the increase in mortality rate on a linear scale than on a logarithmic scale, also when using Gompertz frailty models that account for population heterogeneity.

\section{Acknowledgments}

We thank the patients and the staff of dialysis and transplant units for the contribution of data via their national and regional registries. We thank the following registries for the contribution of data: Austrian Dialysis and Transplant Registry (OEDTR; R. Kramar); Dutch-speaking Belgian Society of Nephrology (NBVN; H. Augustijn and B. De Moor); French-speaking Belgian Society of Nephrology (GNFB; J.M. des Grottes); Danish Nephrology Registry (DNS); Finnish Registry for Kidney Diseases (C. Grönhagen-Riska); The Epidemiology and Information Network in Nephrology (REIN; M. Lassalle); Greek Renal Registry (G.A. Ioannidis); Icelandic End-Stage
Renal Disease Registry; Norwegian Renal Registry (T. Leivestad); Romanian Renal Registry (RRR; G. Mircescu, L. Garneata, and E. Podgoreanu); Slovenian RRT Registry (J. Gubenšek and M. Arnol); Swedish Kidney Registry (SNR; K.G. Prütz, L. Bäckman, M. Evans, S. Schön, M. Stendahl, and B. Rippe); Dutch End-Stage Renal Disease Registry (RENINE; A. Hoitsma and A. Hemke); UK Renal Registry; Scottish Renal Registry (SRR); and the regional registries of Andalusia (SICATA; P. Castro de la Nuez), Aragon (J.I. Sanchez Miret and J.M. Abad Diez), Asturias (R. Alonso de la Torre, J.R. Quirós, and RERCA Working Group), Basque country (UNIPAR; Á. Magaz, J. Aranzabal, M. Rodrigo, and I. Moina), Cantabria (M. Arias Rodríguez and O. García Ruiz), Castile and León (R. González and C. FernándezRenedo), Castile-La Mancha (G. Gutiérrez Ávila and I. Moreno Alía), Catalonia (RMRC; E. Arcos, J. Comas, and J. Tort), Extremadura (J.M. Ramos Aceitero and M.A. García Bazaga), Galicia (E. Bouzas-Caamaño and J. Sánchez-Ibáñez), Community of Madrid (M. Aparicio de Madre and C. Chamorro Jambrina), and Valencian region (REMRENAL; O. Zurriaga Llorens and M. Ferrer Alamar). We thank the other ERA-EDTA Registry committee members for their advice in the 
analysis and the drafting of this article. We thank the Registry's office for the collection and management of data. The ERA-EDTA Registry is funded by the European Renal Association-European Dialysis and Transplant Association (ERA-EDTA).

This article was written by J.J.E.K., A.K., D.v.H., A.Å., J.B.B., J.B.-P., F.C., C.G.C., P.F., J.G.H., Z.A.M., J.M.J.D.M., R.P., R.S., J.P.T., K.J.J., and H.P. on behalf of the ERA-EDTA Registry which is an official body of the ERA-EDTA. J.J.E.K. and H.P. conceived and designed the study. A.K., A.Å., J.-B.B., J.B.-P., F.C., C.G.C., P.F., J.G.H., Z.A.M., J.M.J.D.M., R.P., R.S., J.P.T., and K.J.J. collected the data. H.P. constructed the frailty models. J.J.E.K., A.K., and H.P. analyzed the data. J.J.E.K., A.K., D.v.H., and H.P interpreted the results. J.J.E.K. drafted the manuscript. All authors provided intellectual contributions, read, commented, and approved the manuscript.

\section{Supplementary data}

Supplementary data related to this article can be found at http:// dx.doi.org/10.1016/j.annepidem.2016.08.010.

\section{References}

[1] Finch CE. Longevity, senescence, and the genome. Chicago: University of Chicago Press; 1990. p. 12-32.

[2] Arking R. The biology of aging: observations and principles. New York: Oxford University Press; 2006. p. 26-53.

[3] Vaupel JW. Biodemography of human ageing. Nature 2010;464(7288):536-42.

[4] Driver C. The Gompertz function does not measure ageing. Biogerontology 2001;2(1):61-5.

[5] Rozing MP, Westendorp RGJ. Parallel lines: nothing has changed? Aging Cell 2008;7(6):924-7.

[6] Koopman JJE, Rozing MP, Kramer A, de Jager DJ, Ansell D, De Meester JMJ, et al. Senescence rates in patients with end-stage renal disease: a critical appraisal of the Gompertz model. Aging Cell 2011;10(2):233-8.

[7] Stenvinkel P, Larsson TE. Chronic kidney disease: a clinical model of premature aging. Am J Kidney Dis 2013;62(2):339-51.

[8] Kooman JP, Kotanko P, Schols AM, Shiels PG, Stenvinkel P. Chronic kidney disease and premature ageing. Nat Rev Nephrol 2014;10(12):732-42.

[9] Yilmaz MI, Sonmez A, Saglam M, Cayci T, Kilic S, Unal HU, et al. A longitudinal study of inflammation, CKD-mineral bone disorder, and carotid atherosclerosis after renal transplantation. Clin J Am Soc Nephrol 2015;10(3):471-9.

[10] Vaupel JW, Manton KG, Stallard E. The impact of heterogeneity in individual frailty on the dynamics of mortality. Demography 1979;16(3):439-54.
[11] Vaupel JW, Yashin AI. Heterogeneity's ruses: some surprising effects of selection on population dynamics. Am Stat 1985;39(3):176-85.

[12] Wienke A. Frailty models in survival analysis. Boca Raton: CRC Press; 2011.

[13] Satayathum S, Pisoni RL, McCullough KP, Merion RM, Wikström B, Levin N et al. Kidney transplantation and wait-listing rates from the international Dialysis Outcomes and Practice Patterns Study (DOPPS). Kidney Int 2005;68(1):330-7.

[14] Hemke AC, Heemskerk MBA, van Diepen M, Weimar W, Dekker FW Hoitsma AJ. Survival prognosis after the start of a renal replacement therapy in the Netherlands: a retrospective cohort study. BMC Nephrol 2013; 14:258.

[15] Pippias M, Stel VS, Abad Diez JM, Afentakis N, Herrero-Calvo JA, Arias M, et al. Renal replacement therapy in Europe: a summary of the 2012 ERA-EDTA Registry Annual Report. Clin Kidney J 2015;8(3):248-61.

[16] Wagner M, Ansell D, Kent DM, Griffith JL, Naimark D, Wanner C, et al. Predicting mortality in incident dialysis patients: an analysis of the United Kingdom Renal Registry. Am J Kidney Dis 2011:57(6):894-902.

[17] Kramer A, Stel VS, Caskey FJ, Stengel B, Elliott RF, Covic A, et al. Exploring the association between macroeconomic indicators and dialysis mortality. Clin J Am Soc Nephrol 2012;7(10):1655-63.

[18] Rothman KJ. Epidemiology: an introduction. New York: Oxford University Press; 2002. p. 134.

[19] Vaupel JW, Carey JR, Christensen K, Johnson TE, Yashin AI, Holm NV, et al Biodemographic trajectories of longevity. Science 1998;280(5365):855-60.

[20] Yashin AI, Vaupel JW, Iachine IA. A duality in aging: the equivalence of mortality models based on radically different concepts. Mech Ageing Dev 1994;74(1-2):1-14.

[21] Yashin AI, Iachine IA, Begun AS. Mathematical modeling: a review. Math Popul Stud 2000;8(4):37-41.

[22] Service PM. Heterogeneity in individual mortality risk and its importance for evolutionary studies of senescence. Am Nat 2000;156(1):1-13.

[23] Pletcher SD, Curtsinger JW. The influence of environmentally induced heterogeneity on age-specific genetic variance for mortality rates. Genet Res 2000;75(3):321-9.

[24] Barbi E, Caselli G, Vallin J. Trajectories of extreme survival in heterogeneous populations. Population 2003;58(1):43-65.

[25] Le Cunff Y, Baudisch A, Pakdaman K. How evolving heterogeneity distributions of resource allocation strategies shape mortality patterns. PLoS Comput Biol 2013;9(1):e1002825.

[26] Koopman JJE, Rozing MP, Kramer A, Abad JM, Finne P, Heaf JG, et al. Calculating the rate of senescence from mortality data: an analysis of data from the ERA-EDTA Registry. J Gerontol A Biol Sci Med Sci 2016;71(4):468-74.

[27] Horiuchi S, Wilmoth JR. Deceleration in the age pattern of mortality at older ages. Demography 1998;35(4):391-412.

[28] Gavrilov LA, Gavrilova NS. Mortality measurement at advanced ages: a study of the Social Security Administration Death Master File. N Am Actuar J 2011;15(3):432-47.

[29] Vaupel JW, Baudisch A, Dölling M, Roach DA, Gampe J. The case for negative senescence. Theor Popul Biol 2004;65(4):339-51.

[30] Koopman JJE. Measuring senescence in human populations. Doctoral thesis Leiden (The Netherlands): Leiden University; 2015. p. 193-203. Available at: http://hdl.handle.net/1887/35764 [accessed 22.07.2016]. 